\title{
LA COMPLEMENTACIÓN ACADÉMICAY LA CALIDAD FORMATIVO - PROFESIONAL DE LOS ESTUDIANTES DE INGENIERÍA DE LA UNJBG DETACNA EN EL AÑO 2012
}

\author{
SUPPLEMENTATION FORMATIVE ACADEMIC AND QUALITY- \\ PROFESSIONAL STUDENTS UNJBG ENGINEERING OFTACNA IN 2012
}

\author{
Dionicio Milton Chávez Muñoz,' Julia Marina Mendoza Gómes²
}

\begin{abstract}
RESUMEN
En esta investigación se planteó, "Determinar la influencia de la complementación académica en la calidad formativo-profesional de los estudiantes de ingeniería de la Universidad Nacional Jorge Basadre Grohmann deTacna, en el año 2012". Haciendo una investigación de campo, transversal y en base a encuestas, se arribó a las siguientes conclusiones: La complementación académica que realizan los estudiantes de ingeniería de la UNJBG es baja y los estudiantes posponen esta actividad de complementación para años superiores de estudio o para cuando finaliza la carrera. Mayormente, las instituciones que brindan complementación son los institutos superiores, de preferencia en idiomas, informática y manejo de software. El área de complementación cubre la necesidad en conocimientos prácticos, a fin de tener una formación integral y acceder a mejores opciones de trabajo. La calidad formativo-profesional de los estudiantes de ingeniería también es regular, tendiendo a ser alta; igualmente la formación en contenidos teóricos es regular, tendiendo a ser alta; en contenidos prácticos resulta regular, tendiendo a ser baja; La formación en saber las actividades de su carrera es regular, tendiendo a ser alta, del mismo modo que la formación en saber actuar. La formación en ética y valores es regular, tendiendo a ser alta. En general, la calidad académica de los estudiantes de ingeniería es regular, tendiendo a ser alta.
\end{abstract}

Palabras Clave: Complementación Académica, Ingenierías, formación integral, Calidad formativo profesional.

\section{ABSTRACT}

This research was raised, "To determine the influence of supplementation on quality academic and professional training of engineering students from the National University of Tacna Jorge Basadre Grohmann, in 2012". Doing field research, transverse and based on surveys, it reached the following conclusions: The academic complementation performing students $U N J B G$ engineering students is low and postpone this for years complementation activity higher study or when finish the race. Mostly, institutions are providing colleges complementation, preferably in languages, computers and software management. The area covers the need for complementary skills, in order to have a comprehensive training and access to better job options. The professional-quality training engineering students also regularly tend to be high; equally theoretical training is regular, tending to be high, in practical content is regularly tend to be low, the training activities namely his career is regular, tending to be high, just as training in how to act. Training in ethics and values is regular, tending to be high. In general, the academic quality of engineering students is regular, tending to be high.

Keywords: Academic Complementation, engineering, comprehensive training, professional training quality.

\section{INTRODUCCIÓN}

El Problema: Es de común conocimiento que la formación académica de los estudiantes en las universidades tiene limitaciones y pueden estar referidas a conocimientos, al uso de metodologías de desempeño, a la práctica de la carrera en el seno de la universidad, durante su formación. Según los estándares nacionales e internacionales, un currículo debe ser desarrollado mediante cursos, asignaturas, seminarios, actividades $\mathrm{y} / \mathrm{o}$ talleres de formación técnica básica, de formación profesional, de formación especializada, de formación propedéutica y talleres de prácticas preprofesionales. Cuando en el desarrollo del currículo no se cubren todas las actividades programadas, el estudiante quedará con déficit en los aspectos no cubiertos.

\footnotetext{
'Licenciado en Matemáticas, Magíster en Docencia Universitaria, Doctor en Educación. Facultad de Ciencias de la UNJBG.

Licenciada en Matemáticas, Magister en Docencia Universitaria. Facultad de Ciencias de la UNJBG.
} 
En el caso de las Escuelas de Ingeniería de la UNJBG, el índice de cumplimiento del currículo tiene valores muy por debajo del 100\%. Todo esto amerita que el estudiante complemente su formación profesional con carreras cortas, cursos de capacitación, práctica de laboratorio, visitas de campo, etc.

\section{Objetivos}

Determinar la influencia de la complementación académica en la calidad formativo-profesional de los estudiantes de ingeniería de la UNJBG de Tacna en el año 2012.

a)Identificar la complementación académica que realizan los estudiantes de ingeniería de la UNJBG.

b) Precisar la calidad formativo-profesional de los estudiantes de ingeniería de la UNJBG.

c)Determinar el grado de relación que existe entre la complementación académica y la calidad formativo-profesional de los estudiantes de ingeniería de la UNJBG.

\section{Justificación}

Los resultados de la presente investigación permitirán a los directivos de las escuelas profesionales es conocer que el estudiante necesita complementar su carrera con otras afines. El estudiante sabrá de las falencias que tiene durante su formación para ir complementando, según su orientación laboral. La Escuela Profesional puede apoyar en la capacitación.

\section{MATERIALES Y MÉTODOS}

La investigación se realizó mediante un estudio descriptivo correlacional, con diseño transversal y aplicación de encuestas y test a 407 estudiantes de las diferentes escuelas de ingeniería de la UNJBG.

\section{RESULTADOS}

\section{A. Complementación académica.}

Tabla $\mathrm{N}^{\circ}$ 01: Distribución de estudiantes por facultades según si han hecho otros estudios.

\begin{tabular}{|c|c|c|c|c|c|c|c|c|}
\hline \multirow{2}{*}{$\begin{array}{c}\text { Ha } \\
\text { comple- } \\
\text { mentado } \\
\text { con } \\
\text { otros } \\
\text { estudios }\end{array}$} & \multicolumn{5}{|c|}{$\begin{array}{c}\text { DISTRIBUCIÓN DE } \\
\text { ESTUDIANTES POR }\end{array}$} & \multicolumn{3}{c|}{ Total } \\
\cline { 2 - 8 } & $\mathbf{n}$ & $\%$ & $\mathbf{n}$ & $\%$ & $\mathbf{n}$ & $\%$ & $\mathbf{n}$ & $\%$ \\
\hline FA & 115 & 49,6 & 47 & 48,0 & 30 & 39,0 & 192 & 47,2 \\
\hline SI & 117 & 50,4 & 51 & 52,0 & 47 & 61,0 & 215 & 52,8 \\
\hline Total & 282 & 100 & 98 & 100 & 77 & 100 & 407 & 100 \\
\hline
\end{tabular}

Fuente: Encuesta aplicada a estudiantes.
Tabla $\mathrm{N}^{\circ}$ 02: Distribución según si estudia en instituto técnico.

\begin{tabular}{|c|c|c|c|c|c|c|c|c|}
\hline \multirow[t]{3}{*}{ Estudia } & \multicolumn{6}{|c|}{$\begin{array}{c}\text { DISTRIBUCIÓN DE } \\
\text { ESTUDIANTES POR } \\
\text { FACULTADES }\end{array}$} & \multirow{2}{*}{\multicolumn{2}{|c|}{ Total }} \\
\hline & \multicolumn{2}{|c|}{ FAIN } & \multicolumn{2}{|c|}{ FCAG } & \multicolumn{2}{|c|}{ FIAG } & & \\
\hline & $\mathrm{n}$ & $\%$ & $\mathrm{n}$ & $\%$ & $n$ & $\%$ & $n$ & $\%$ \\
\hline No & 155 & 66,8 & 84 & 85,7 & 43 & 55,8 & 282 & 69,3 \\
\hline SI & 77 & 33,2 & 14 & 14,3 & 34 & 44,2 & 125 & 30,7 \\
\hline Total & 232 & 100 & 98 & 100 & 77 & 100 & 407 & 100 \\
\hline
\end{tabular}

Fuente: Encuesta aplicada a estudiantes.

Tabla $\mathrm{N}^{\circ}$ 03: Distribución según si estudia en centro de idiomas.

\begin{tabular}{|c|c|c|c|c|c|c|c|c|}
\hline \multirow{3}{*}{ Estudia } & \multicolumn{6}{|c|}{$\begin{array}{c}\text { DISTRIBUCIÓN DE } \\
\text { ESTUDIANTES POR } \\
\text { FACULTADES }\end{array}$} & \multirow{2}{*}{\multicolumn{2}{|c|}{ Total }} \\
\hline & \multicolumn{2}{|c|}{ FAIN } & \multicolumn{2}{|c|}{ FCAG } & \multicolumn{2}{|c|}{ FIAG } & & \\
\hline & $n$ & $\%$ & $n$ & $\%$ & $n$ & $\%$ & $n$ & $\%$ \\
\hline No & 132 & 56,9 & 54 & 55,1 & 54 & 70,1 & 240 & 59,0 \\
\hline SI & 100 & 43,1 & 44 & 44,9 & 23 & 29,9 & 167 & 41,0 \\
\hline Total & 232 & 100 & 98 & 100 & 77 & 100 & 407 & 100 \\
\hline
\end{tabular}

Fuente: Encuesta aplicada a estudiantes.

Con lo cual se observa que los estudiantes de la UNJBG realizan complementación académica de sus carreras.

\section{B. Carrera de complementación}

Tabla $\mathrm{N}^{\circ}$ 04: Distribución según si estudia una carrera técnica.

\begin{tabular}{|c|c|c|c|c|c|c|c|c|}
\hline \multirow[t]{3}{*}{ Estudia } & \multicolumn{6}{|c|}{$\begin{array}{l}\text { DISTRIBUCION DE } \\
\text { ESTUDIANTES POR } \\
\text { FACULTADES }\end{array}$} & \multirow{2}{*}{\multicolumn{2}{|c|}{ Total }} \\
\hline & \multicolumn{2}{|c|}{ FAIN } & \multicolumn{2}{|c|}{ FCAG } & \multicolumn{2}{|c|}{ FIAG } & & \\
\hline & $\mathrm{n}$ & $\%$ & $n$ & $\%$ & $\mathrm{n}$ & $\%$ & $\mathrm{n}$ & $\%$ \\
\hline NO & 160 & 69,0 & 72 & 79,5 & 53 & 68,8 & 28. & 70,0 \\
\hline SI & 72 & 31,0 & 26 & 26,5 & 24 & 31,2 & 129 & 30,0 \\
\hline Total & 232 & 100 & 98 & 100 & 77 & 100 & 407 & 100 \\
\hline
\end{tabular}

Fuente: Encuesta aplicada a estudiantes.

Tabla $\mathrm{N}^{\circ}$ 05: Distribución según si estudia manejo de software de ingeniería.

\begin{tabular}{|c|c|c|c|c|c|c|c|c|}
\hline \multirow{3}{*}{ Estudia } & \multicolumn{6}{|c|}{$\begin{array}{l}\text { DISTRIBUCIÓN DE } \\
\text { ESTUDIANTES POR } \\
\text { FACULTADES }\end{array}$} & \multirow{2}{*}{\multicolumn{2}{|c|}{ Total }} \\
\hline & \multicolumn{2}{|c|}{ FAIN } & \multicolumn{2}{|c|}{ FCAG } & \multicolumn{2}{|c|}{ FIAG } & & \\
\hline & $\mathrm{n}$ & $\%$ & $\mathrm{n}$ & $\%$ & $\mathrm{n}$ & $\%$ & $n$ & $\%$ \\
\hline NO & 146 & 62,9 & 86 & 87,8 & 49 & 55,8 & 27.5 & 67,6 \\
\hline SI & 86 & 37,1 & 12 & 12,2 & 34 & 44,2 & 132 & 32,4 \\
\hline Total & 232 & 100 & 98 & 100 & 77 & 100 & 407 & 100 \\
\hline
\end{tabular}

Fuente: Encuesta aplicada a estudiantes. 
Tabla $\mathrm{N}^{\circ}$ 06: Distribución según si estudia otro idioma.

\begin{tabular}{|c|c|c|c|c|c|c|c|c|}
\hline \multirow[t]{3}{*}{ Estudia } & \multicolumn{6}{|c|}{$\begin{array}{l}\text { DISTRIBUCIÓN DE } \\
\text { ESTUDIANTES POR } \\
\text { FACULTADES }\end{array}$} & \multirow{2}{*}{\multicolumn{2}{|c|}{ Total }} \\
\hline & \multicolumn{2}{|c|}{ FAIN } & \multicolumn{2}{|c|}{ FCAG } & \multicolumn{2}{|c|}{ FIAG } & & \\
\hline & $\mathrm{n}$ & $\%$ & $\mathrm{n}$ & $\%$ & $\mathrm{n}$ & $\%$ & n & $\%$ \\
\hline NO & 132 & 56,9 & 53 & 54,1 & 56 & 72,7 & 241 & 59,2 \\
\hline SI & 100 & 43,1 & 45 & 45,9 & 21 & 27,3 & 166 & 40,8 \\
\hline Total & 282 & 100 & 98 & 100 & 77 & 100 & 407 & 100 \\
\hline
\end{tabular}

Fuente: Encuesta aplicada a estudiantes.

Con lo que se verifica que el estudiante se complementa con carreras técnicas, manejo de software y otros idiomas.

\section{Razones para complementar la carrera}

Tabla $\mathrm{N}^{\circ}$ 07: Distribución según que estudia para realizar más prácticas profesionales.

\begin{tabular}{|c|c|c|c|c|c|c|c|c|}
\hline \multirow[t]{3}{*}{ Estudia } & \multicolumn{6}{|c|}{$\begin{array}{c}\text { DISTRIBUCION DE } \\
\text { ESTUDIANTES POR } \\
\text { FACULTADES }\end{array}$} & \multirow{2}{*}{\multicolumn{2}{|c|}{ Total }} \\
\hline & \multicolumn{2}{|c|}{ FAIN } & \multicolumn{2}{|c|}{ FCAG } & \multicolumn{2}{|c|}{ FIAG } & & \\
\hline & $\mathrm{n}$ & $\%$ & $\mathrm{n}$ & $\%$ & $\mathrm{n}$ & $\%$ & $\mathrm{n}$ & $\%$ \\
\hline NO & 100 & 43,1 & 51 & 52,0 & 29 & 37,7 & 180 & 44,2 \\
\hline SI & 132 & 56,9 & 47 & 48,0 & 48 & 62,3 & 297 & 55,8 \\
\hline Total & 232 & 100 & 98 & 100 & 77 & 100 & 407 & 100 \\
\hline
\end{tabular}

Fuente: Encuesta aplicada a estudiantes.

Tabla $\mathrm{N}^{\circ}$ 08: Distribución según que estudia para llenar vacíos teóricos.

\begin{tabular}{|c|c|c|c|c|c|c|c|c|}
\hline \multirow[t]{3}{*}{ Estudia } & \multicolumn{6}{|c|}{$\begin{array}{c}\text { DISTRIBUCIÓN DE } \\
\text { ESTUDIANTES POR } \\
\text { FACULTADES }\end{array}$} & \multirow{2}{*}{\multicolumn{2}{|c|}{ Total }} \\
\hline & \multicolumn{2}{|c|}{ FAIN } & \multicolumn{2}{|c|}{ FCAG } & \multicolumn{2}{|c|}{ FIAG } & & \\
\hline & $n$ & $\%$ & $\mathrm{n}$ & $\%$ & n & $\%$ & $\mathrm{n}$ & $\%$ \\
\hline No & 169 & 72,8 & 68 & 69,4 & 47 & 61,0 & 284 & 69,8 \\
\hline SI & 63 & 27,2 & 30 & 30,6 & 30 & 39,0 & 123 & 30,2 \\
\hline Total & 232 & 100 & 98 & 100 & 77 & 100 & 407 & 100 \\
\hline
\end{tabular}

Fuente: Encuesta aplicada a estudiantes.

Tabla $\mathrm{N}^{\circ}$ 09: Distribución según que estudia para realizar trabajo de campo.

\begin{tabular}{|c|c|c|c|c|c|c|c|c|}
\hline \multirow{3}{*}{ Estudia } & \multicolumn{5}{|c|}{$\begin{array}{c}\text { DISTRIBUCIÓN DE } \\
\text { ESTUDIANTES POR }\end{array}$} & \multicolumn{3}{c|}{ Total } \\
\cline { 2 - 8 } & \multicolumn{3}{|c|}{ FACULTADES } & \multicolumn{2}{c|}{} \\
\cline { 2 - 8 } & $\mathbf{n}$ & $\%$ & $\mathrm{n}$ & $\%$ & $\mathrm{n}$ & $\%$ & $\mathbf{n}$ & $\%$ \\
\hline NO & 192 & 52,6 & 61 & 62,2 & 38 & 49,4 & 221 & 54,5 \\
\hline SI & 110 & 47,4 & 37 & 37,8 & 39 & 50,6 & 186 & 45,7 \\
\hline Total & 292 & 100 & 98 & 100 & 77 & 100 & 407 & 100 \\
\hline
\end{tabular}

Fuente: Encuesta aplicada a estudiantes.
Tabla $\mathrm{N}^{\circ}$ 10: Distribución según que estudia para tener más opciones de trabajo.

\begin{tabular}{|c|c|c|c|c|c|c|c|c|}
\hline \multirow{3}{*}{ Estudia } & \multicolumn{5}{|c|}{$\begin{array}{c}\text { DISTRIBUCION DE } \\
\text { ESTUDIANTES POR } \\
\text { FACULTADES }\end{array}$} & \multicolumn{3}{c|}{ Total } \\
\cline { 2 - 9 } & \multicolumn{3}{|c|}{ FAIN } & \multicolumn{2}{|c|}{ FCAG } & \multicolumn{2}{c|}{ FIAG } & \multicolumn{2}{c|}{} \\
\cline { 2 - 9 } & $\mathbf{n}$ & $\%$ & $\mathbf{n}$ & $\%$ & $\mathbf{n}$ & $\%$ & $\mathbf{n}$ & $\%$ \\
\hline NO & 89 & 38,4 & 46 & 46,9 & 32 & 41,6 & 167 & 41,0 \\
\hline SI & 149 & 61,6 & 52 & 53,1 & 45 & 58,4 & 240 & 59,0 \\
\hline Total & 292 & 100 & 98 & 100 & 77 & 100 & 407 & 100 \\
\hline
\end{tabular}

Fuente: Encuesta aplicada a estudiantes.

Tabla $\mathrm{N}^{\circ}$ 11: Distribución según que estudia para tener formación integral.

\begin{tabular}{|c|c|c|c|c|c|c|c|c|}
\hline \multirow[t]{3}{*}{ Estudia } & \multicolumn{6}{|c|}{$\begin{array}{c}\text { DISTRIBUCIÓN DE } \\
\text { ESTUDIANTES POR } \\
\text { FACULTADES }\end{array}$} & \multirow{2}{*}{\multicolumn{2}{|c|}{ Total }} \\
\hline & \multicolumn{2}{|c|}{ FAIN } & \multicolumn{2}{|c|}{ FCAG } & \multicolumn{2}{|c|}{ FIAG } & & \\
\hline & $\mathrm{n}$ & $\%$ & $n$ & $\%$ & $\mathrm{n}$ & $\%$ & $\mathrm{n}$ & $\%$ \\
\hline No & 107 & 46,1 & 50 & 51,0 & 30 & 39,9 & 187 & 45,9 \\
\hline SI & 125 & 53,9 & 48 & 49,0 & 47 & 61,0 & 220 & 54,1 \\
\hline Total & 232 & 100 & 98 & 100 & 77 & 100 & 407 & 100 \\
\hline
\end{tabular}

Fuente: Encuesta aplicada a estudiantes.

Observamos que el estudiante complementa su carrera para realizar más prácticas, para llenar vacíos teóricos, para realizar trabajo de campo, y para tener una formación integral.

\section{Resultados de la variable dependiente: Calidad formativo profesional}

Tabla $\mathrm{N}^{\circ}$ 12: Distribución según la formación en conocimientos teóricos.

\begin{tabular}{|c|c|c|c|c|c|c|c|c|}
\hline & \multicolumn{6}{|c|}{$\begin{array}{l}\text { DISTRIBUCIÓN DE ESTUDIANTES } \\
\text { POR FACULTADES }\end{array}$} & \multirow{2}{*}{\multicolumn{2}{|c|}{ Total }} \\
\hline & \multicolumn{2}{|c|}{ FAIN } & \multicolumn{2}{|c|}{ FCAG } & \multicolumn{2}{|c|}{ FIAG } & & \\
\hline & $n$ & $\%$ & $\mathrm{n}$ & $\%$ & $n$ & $\%$ & $\mathrm{n}$ & $\%$ \\
\hline $\begin{array}{l}\text { MUY } \\
\text { BAJO }\end{array}$ & 4 & 1,7 & 1 & 1,0 & s & 8,9 & 8 & 2,0 \\
\hline BAJO & 12 & 5,2 & 8 & 8,2 & 4 & 5,2 & 24 & 5,9 \\
\hline REGULAR & 97 & 41,8 & 49 & 50.0 & $\$ 7$ & 18,1 & 183 & 45,0 \\
\hline ALTO & 107 & 46,1 & 37 & 37.8 & 31 & $\leftrightarrow 0,5$ & 175 & 43,0 \\
\hline $\begin{array}{l}\text { MUY } \\
\text { ALTO }\end{array}$ & 12 & 5,2 & $s$ & $s, 1$ & 2 & 2,6 & 17 & k.2 \\
\hline Total & 232 & 100 & 98 & 100 & 77 & 100 & 407 & 100 \\
\hline
\end{tabular}

Fuente: Encuesta aplicada a estudiantes.

Tabla $\mathrm{N}^{\circ}$ 13: Distribución según la formación en conocimientos prácticos.

\begin{tabular}{|c|c|c|c|c|c|c|c|c|}
\hline \multirow{2}{*}{} & \multicolumn{5}{|c|}{$\begin{array}{c}\text { DISTRIBUCIÓN DE } \\
\text { ESTUDIANTES POR } \\
\text { FACULTADES }\end{array}$} & \multicolumn{3}{c|}{ Total } \\
\cline { 2 - 9 } & \multicolumn{3}{|c|}{ FAIN } & \multicolumn{2}{|c|}{ FCAG } & \multicolumn{2}{c|}{ FIAG } & \multicolumn{2}{c|}{} \\
\cline { 2 - 9 } & $\mathrm{n}$ & $\%$ & $\mathrm{n}$ & $\%$ & $\mathrm{n}$ & $\%$ & $\mathrm{n}$ & $\%$ \\
\hline $\begin{array}{c}\text { MUY } \\
\text { BAJO }\end{array}$ & 20 & 8,6 & 13 & 13,3 & 12 & 15,6 & 45 & 11,1 \\
\hline BAJO & 50 & 21,6 & 31 & 31,6 & 24 & 31,2 & 105 & 25,8 \\
\hline REGULAR & 107 & 46,1 & 42 & 49,9 & 82 & 41,6 & 181 & 45,5 \\
\hline ALTO & 47 & 20,3 & 12 & 12,2 & 9 & 11,7 & 68 & 16,7 \\
\hline $\begin{array}{c}\text { MUY } \\
\text { ALTO }\end{array}$ & 8 & 5,4 & 0 & 0,0 & 0 & 0,0 & 8 & 2,0 \\
\hline Total & 232 & 100 & 98 & 100 & 77 & 100 & 407 & 100 \\
\hline
\end{tabular}

Fuente: Encuesta aplicada a estudiantes. 
Tabla $\mathrm{N}^{\circ}$ 14: Distribución según la formación para el desempeño profesional en saber hacer las cosas.

\begin{tabular}{|c|c|c|c|c|c|c|c|c|}
\hline \multirow[t]{3}{*}{ 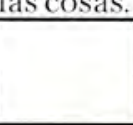 } & \multicolumn{6}{|c|}{$\begin{array}{l}\text { DISTRIBUCIÓN DE ESTUDIANTES } \\
\text { POR FACULTADES }\end{array}$} & \multirow{2}{*}{\multicolumn{2}{|c|}{ Total }} \\
\hline & \multicolumn{2}{|c|}{ FAIN } & \multicolumn{2}{|c|}{ FCAG } & \multicolumn{2}{|c|}{ FLAG } & & \\
\hline & $\mathrm{n}$ & $\%$ & $n$ & $\%$ & $n$ & $\%$ & $n$ & $\%$ \\
\hline $\begin{array}{l}\text { MUY } \\
\text { BAJO }\end{array}$ & 4 & 1,7 & 1 & 1,0 & 1 & 1,3 & 6 & 1,5 \\
\hline BAJO & 18 & 7.8 & 9 & 9.9 & 6 & 7,8 & 33 & 8,1 \\
\hline REGULAR & 112 & 48,3 & 56 & 57,1 & +2 & $5 \hbar, 5$ & 210 & 51,6 \\
\hline ALTO & 91 & 39,2 & 32 & 32,7 & 26 & 33,8 & 149 & 36,6 \\
\hline $\begin{array}{l}\text { MUY } \\
\text { ALTO }\end{array}$ & 7 & $\mathrm{~s}, \mathrm{o}$ & 0 & 0,0 & 2 & 2,6 & 9 & 2,2 \\
\hline Total & 232 & 100 & 98 & 100 & 77 & 100 & 607 & 100 \\
\hline
\end{tabular}

Fuente: Encuesta aplicada a estudiantes.

Tabla $\mathrm{N}^{\circ}$ 15: Distribución según la formación para el desempeño profesional en saber actuar.

\begin{tabular}{|c|c|c|c|c|c|c|c|c|}
\hline & \multicolumn{6}{|c|}{$\begin{array}{l}\text { DISTRIBUCIÓN DE } \\
\text { ESTUDIANTES POR } \\
\text { FACULTADES }\end{array}$} & \multirow{2}{*}{\multicolumn{2}{|c|}{ Total }} \\
\hline & \multicolumn{2}{|c|}{ FAIN } & \multicolumn{2}{|c|}{$\mathrm{FCAG}$} & \multicolumn{2}{|c|}{ FIAG } & & \\
\hline & $n$ & $\%$ & $n$ & $\%$ & $\mathrm{n}$ & $\%$ & $n$ & $\%$ \\
\hline $\begin{array}{l}\text { MUY } \\
\text { BAJOO }\end{array}$ & 5 & 2,2 & 5 & 5,1 & 2 & 2,6 & 12 & 2.9 \\
\hline BAJO & 37 & 15,9 & 10 & 10,2 & 10 & 13,0 & 57 & 14,0 \\
\hline REGULAR & 107 & 46,1 & 61 & 62,2 & +2 & 54,5 & 210 & 51,6 \\
\hline ALTO & 74 & 31,9 & 22 & 22,4 & 20 & 26,0 & 116 & 28,5 \\
\hline $\begin{array}{l}\text { MUY } \\
\text { ALTO } \\
\end{array}$ & 9 & $\$ 9$ & 0 & 0,0 & * & 3,9 & 12 & 2,9 \\
\hline Total & 232 & 100 & 98 & 100 & 77 & 100 & 407 & 100 \\
\hline
\end{tabular}

Fuente: Encuesta aplicada a estudiantes.

Tabla N 16: Distribución según la formación en actitudes: ética y valores.

\begin{tabular}{|c|c|c|c|c|c|c|c|c|}
\hline & \multicolumn{6}{|c|}{$\begin{array}{l}\text { DISTRIBUCION DE ESTUDIANTES } \\
\text { POR FACULTADES }\end{array}$} & \multirow{2}{*}{\multicolumn{2}{|c|}{ Total }} \\
\hline & \multicolumn{2}{|c|}{ FAIN } & \multicolumn{2}{|c|}{$\mathrm{FCAG}$} & \multicolumn{2}{|c|}{ FIAG } & & \\
\hline & $n$ & $\%$ & $\mathrm{n}$ & $\%$ & $\mathrm{n}$ & $\%$ & $n$ & $\%$ \\
\hline $\begin{array}{l}\text { MUY } \\
\text { BAJO }\end{array}$ & 5 & 2,2 & 3 & 3,1 & 2 & 2,6 & 10 & 2.5 \\
\hline BAJO & 37 & 15,9 & 17 & 17,3 & 13 & 16,9 & 67 & 16,5 \\
\hline REGULAR & 99 & $+2,7$ & 42 & 42,9 & 39 & 50,6 & 180 & $\mathrm{H}, 2$ \\
\hline ALTO & 80 & 34,5 & 35 & $\$ 5.7$ & 21 & 27.3 & 136 & 33,4 \\
\hline $\begin{array}{l}\text { MUY } \\
\text { ALTO }\end{array}$ & 11 & +.7 & t & 1.0 & 2 & 2,6 & $1+$ & 3.4 \\
\hline Total & 232 & 100 & 98 & 100 & 77 & 100 & 407 & 100 \\
\hline
\end{tabular}

Fuente: Encuesta aplicada a estudiantes.

Tabla $\mathrm{N}^{\circ}$ 17: Distribución según la formación profesional recibida en su facultad y escuela profesional.

\begin{tabular}{|c|c|c|c|c|c|c|c|c|}
\hline & \multicolumn{6}{|c|}{$\begin{array}{l}\text { DISTRIBUCION DE ESTUDIANTES } \\
\text { POR FACULTADES }\end{array}$} & \multirow{2}{*}{\multicolumn{2}{|c|}{ Total }} \\
\hline & \multicolumn{2}{|c|}{ FAIN } & \multicolumn{2}{|c|}{ FCAG } & \multicolumn{2}{|c|}{ FIAG } & & \\
\hline & $n$ & $\%$ & $\bar{n}$ & $\%$ & $n$ & $\%$ & $n$ & $\%$ \\
\hline $\begin{array}{l}\text { MUY } \\
\text { BAJO }\end{array}$ & 2 & 0,9 & 5 & 5,1 & 1 & 1,3 & 8 & 2,0 \\
\hline BAJO & 19 & 8,2 & 5 & 5,1 & 6 & 7,8 & 30 & 7,4 \\
\hline REGULAR & $10 \mathrm{H}$ & $+1, \mathrm{~S}$ & 56 & 57,1 & 38 & $+9,4$ & 198 & 48,6 \\
\hline ALTO & 95 & 40.1 & 32 & 32.7 & so & $\$ 9,0$ & 155 & 38,1 \\
\hline $\begin{array}{l}\text { MUY } \\
\text { ALTO }\end{array}$ & 14 & 6,0 & 0 & 0,0 & $g$ & 2,6 & 16 & 5,9 \\
\hline Total & 232 & 100 & 98 & 100 & 77 & 100 & 407 & 100 \\
\hline
\end{tabular}

Fuente: Encuesta aplicada a estudiantes.

Se observa que la calidad formativa del estudiante se ubica entre regular y alta.

\section{DISCUSIÓN}

Análisis preliminar de los resultados de la investigación

\subsection{Variable independiente: La complementación académica}

La manifestación de los estudiantes respecto a su complementación académica es bastante baja, dado que son pocos los que tienen la intención de realizarlo posteriormente. Conocido es que ante un mundo laboral competitivo, el profesional, al egresar de la universidad, tiene que enfrentar competencias que muchas veces no les ha brindado o les ha brindado muy poco; entonces sus posibilidades de insertarse al mundo laboral se reducen; es por esta razón que la complementación académica para los egresados se torna importante y obligatoria. Que un profesional tenga amplias y diversas competencias lo coloca en mejores condiciones para iniciarse en su desempeño laboral.

La complementación puede ser asumida por la universidad, escuela profesional, otra institución particular o por iniciativa del estudiante, inclusive, durante la formación profesional o al culminar la carrera. Existe una gama de áreas de capacitación, destacando: estudios en software de ingeniería y de idiomas, con la visión de tener una formación integral.

\section{Respecto a la necesidad de complementar la carrera:}

Más del $50 \%$ de los estudiantes está haciendo estudios para complementar su carrera. Este hecho es un indicador que la formación que recibe en la universidad necesita ser completada para quedar expedito de cara al mercado laboral.

\section{Respecto a la carrera de complementación:}

En mayor medida, el estudiante complementaría su formación profesional estudiando una carrera que va por la línea de la informática, sobre todo software de aplicación en la ingeniería que estudia.

El manejo de software está bastante considerado como actividad de complementación de las carreras de la universidad.

Estudiar un idioma está entre las prioridades de complementación, sobre todo, para seguir estudiando bibliografía que está en otros idiomas o para posteriores estudios de postgrado en el país o en el extranjero.

Respecto a las razones para complementar la carrera: 
Dentro de las razones para estudiar otra carrera y complementar su especialidad universitaria está el refuerzo de las prácticas profesionales. Como se sabe, la universidad forma a los profesionales más en conocimientos teóricos que en prácticas, por lo que opta por esta opción de mejora, dado que el desempeño laboral tiene una alta actividad técnica.

Otra razón importante para complementar la carrera profesional es la oportunidad de realizar trabajo de campo. Estar mejor capacitado equivale a ampliar el abanico de opciones de trabajo en el mercado laboral.

\subsection{Variable dependiente: Calidad formativo profesional}

La calidad formativo-profesional está mayoritariamente expresada por el nivel regular, tanto en conocimientos teóricos como en conocimientos prácticos, en formación procedimental y en actitudes y valores, en saber hacer las cosas de nuestra profesión y en saber actuar. En algunos aspectos particulares se matizan con niveles bajos como en el caso de formación en aspectos prácticos y en otros se matizan con niveles altos como en los casos de saber hacer las cosas en su profesión y en formación ética y en valores; lo cual quiere decir que nuestra universidad tiene sus limitaciones pero que no son abismales, sino limitaciones que van por la línea del desarrollo de las ciencias y al adelanto natural de las carreras profesionales.

Observando la calidad formativo profesional, en general, está en un nivel regular tendiendo a un nivel alto.

\section{CONCLUSIONES}

- La complementación académica que realizan los estudiantes de ingeniería de la UNJBG de Tacna es baja y los estudiantes la posponen, en mayor medida, para años superiores de estudio o para cuando finalice la carrera. Mayormente los lugares de complementación corresponden a los institutos superiores y a los de idiomas, informática y manejo de software. El área de complementación es en conocimientos prácticos; $\mathrm{y}$, una razón es para tener una formación integral y acceder a mejores opciones de trabajo.

- La calidad formativo-profesional de los estudiantes de ingeniería de la universidad Nacional Jorge Basadre Grohmann de Tacna es regular, tendiendo a ser alta.

- La formación en contenidos teóricos es regular tendiendo a ser alta; en contenidos prácticos es regular tendiendo a ser baja.
- La formación en saber las cosas de su carrera es regular, tendiendo a ser alta, del mismo modo pasa con la formación en saber actuar en su carrera.

- La formación en ética y valores es regular, tendiendo a ser alta.

- En general la calidad académica de los estudiantes de ingeniería es regular, tendiendo a ser alta.

\section{REFERENCIAS BIBLIOGRÁFICAS}

Alvarado Oyarse, Otoniel (1999) "Política Educativa: conceptos, reflexiones y propuestas". Universidad Cesar vallejo. Trujillo-Perú.

Barrientos Jimenez, Elsa (2000) "Egresado en Educación y su relación con el perfil curricular y el curriculum". Lima-Perú.

Biencinto López, Chantal (2003) "Evaluación del impacto de la formación continua en el ámbito sanitario: Diseño y especificación de un modelo casual". Universidad Complutense de Madrid. Madrid, España.

Calderón Franco, Martha (2002) "Calidad de formación profesional de los alumnos de obstetricia en la UNMSM y la UNSCH". Lima. Perú.

De Zubiria, Miguel (1994) "Tratado de Pedagogía Conceptual. Pensamiento y aprendizaje". Colombia.

Estrada Rivera, Marvin David (2010) "Calidad Profesional en el estudiante egresado de la Carrera de Mecánica Automotriz del Instituto Técnico Industrial del Municipio del Asintal, Departamento de Retalhuleu". Universidad panamericana. Guatemala.

Kennth Delgado (1995) "Evaluación y Calidad de la Educación”. Editorial LOGO. Lima-Perú.

Peñaloza Ramelia, Walter (2003): Los Propósitos de la Educación. Fondo Editorial del Pedagógico San Marcos. Lima-Perú.

Planes curriculares de las Escuelas Académico Profesionales de la UNJBG de Tacna.

\section{Correspondencia:}

Dinicio Milton Chavez Muñoz

Ciudad Universitaria fundo "Los Granados"

Av. Miraflores s/n Tacna - Perú

Julia Marina Mendoza Gómes

Ciudad Universitaria fundo "Los Granados"

Av. Miraflores s/n Tacna - Perú 